
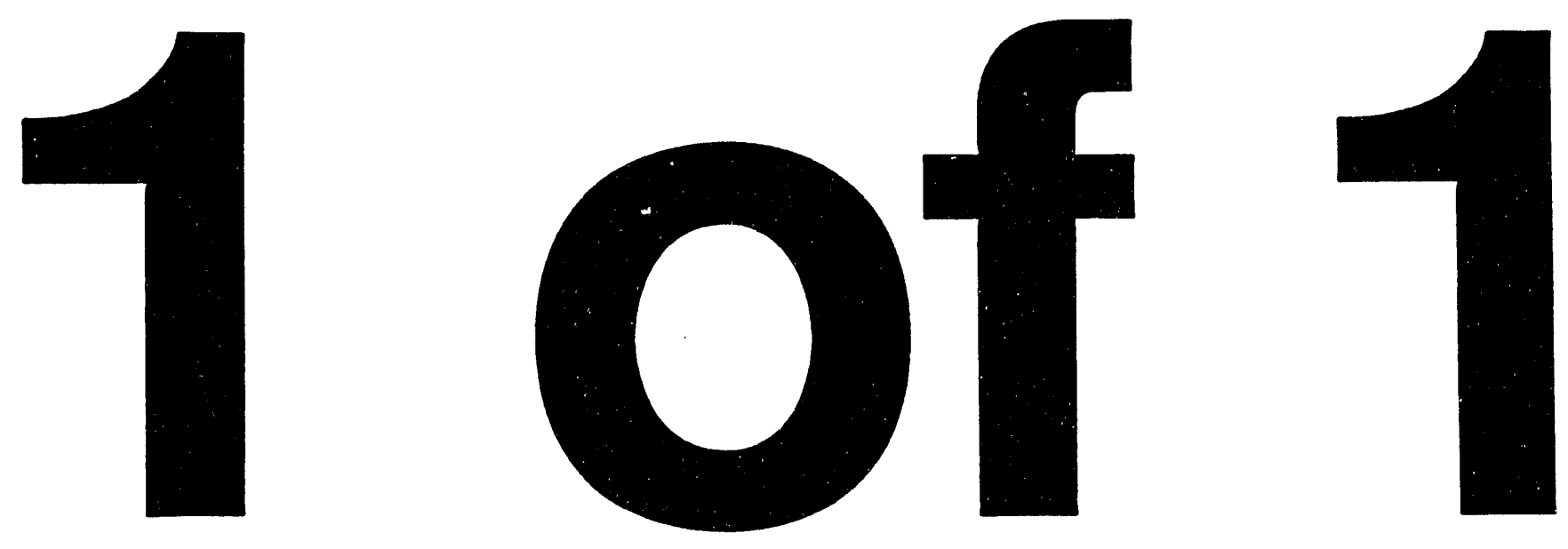


\title{
Universal Features of Multiplicity Distributions
}

\author{
A. B. Balantekin \\ Physics Department, University of Wisconsin, Madison, Wisconsin, 53706 \\ Institute for Nuclear Theory, University of Washington, Seattle, Washington, 98195
}

\section{PREPARED FOR THE U.S. DEPARTMENT OF ENERGY UNDER GRANT DE-FG06-90ER40561}

This report was prepared as an account of work sponsored by the United States Government. Neither the United States nor ?ny agency thereof, nor any of their employees, makes any warranty, express or implied, or assumes any legal liability or responsibility for the accuracy, completeness, or usefulness of any information, apparatus, product, or process disclosed, or represents that its use would not infringe privately owned rights. Reference herein to any specific commercial product, process, or service by trade name, mark, manufacturer, or otherwise, does not necessarily constitute or imply its endorsement, recommendation, or favoring by the United States Government or any agency thereof. The views and opinions of authors expressed herein do not necessarily state or reflect those of the United States Government or any agency thereof.

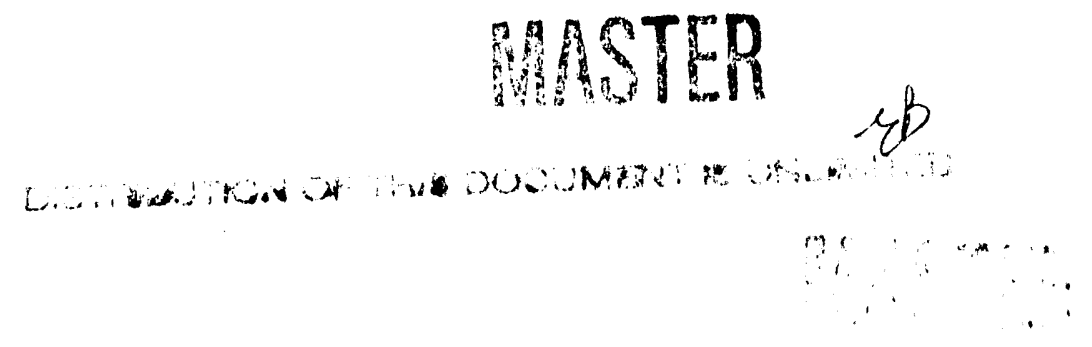




\title{
Universal Features of Multiplicity Distributions
}

\author{
A.B. BALANTEKIN \\ Physics Department, University of Wisconsin, \\ Madison, Wisconsin, 53706 USA \\ and \\ Institute for Nuclear Theory, University of Washington \\ Seattle, WA 98195 USA
}

\begin{abstract}
Universal features of multiplicity distributions are studied and combinants, certain linear combinations of ratios of probabilities, are introduced. It is argued that they can be a useful tool in analyzing multiplicity distributions of hadrons emitted in high energy collisions and large scale structure of galaxy distributions.
\end{abstract}

\section{Introduction}

To understand the nature of the deconfinement transition in ultrarelativistic heavy ion collisions one would like to study particle correlations in the coordinate space. Experimentally one can only measure these correlations in momentum space. To fruitfully utilize correlation measurements as a clean signal of phase seperation, it is necessary to identify particles coming from the decays of resonances. A preliminary investigation of the effects of decays and final-state interactions on velocity correlations was given in [1]. In this talk we first discuss possible ways of distinguishing between particles coming from the decay of some resonance and those originating elsewhere and then elaborate on the universal features of multiplicity distributions.

Multiplicity distributions in high energy heavy-ion and hadron collisions were measured for a wide range of energies. The set of probabilities $P_{N}=$ 
$\sigma_{N} /\left(\sum_{N} \sigma_{N}\right)$, where $\sigma_{N}$ is the cross-section for producing $N$ particles of a given kind, provide information about the production mechanism. Simple models for boson production, such as emission from classical currents [2], predict that the multiplicity distribution is Poisson, the generating function of which is given by

$$
F(\lambda)=\sum_{N=0}^{\infty} P_{N} \lambda^{N}=\exp [(\lambda-1)\langle N\rangle]
$$

where $\langle N\rangle$ is the mean multiplicity. Experimental observation of Poisson distributions indicate that the emitted bosons are uncorrelated in momentum space. In order to investigate deviations from the Poisson distribution, Kauffmann and Gyulassy introduced [3] combinants, $\mathcal{C}_{N}$. They are defined via the expansion

$$
\log F(\lambda)=\log P_{0}+\sum_{k=1}^{\infty} \mathcal{C}_{k} \lambda^{k}
$$

For the Poisson distribution $\mathcal{C}_{1}=\langle n\rangle, \mathcal{C}_{2}=\mathcal{C}_{3}=\ldots=0$. Each combinant $\mathcal{C}_{k}$ is expressible as a combination of just the first $k$ probability ratios (hence the name), which is a potential advantage over the usual cumulants. One can easily show that

$$
\begin{gathered}
\mathcal{C}_{1}=P_{1} / P_{0} \\
\mathcal{C}_{2}=\left(P_{2} / P_{0}\right)-\frac{1}{2}\left(P_{1} / P_{0}\right)^{2}, \\
\mathcal{C}_{3}=\left(P_{3} / P_{0}\right)-\left(P_{1} / P_{0}\right)\left(P_{2} / P_{0}\right)+\frac{1}{3}\left(P_{1} / P_{0}\right)^{3},
\end{gathered}
$$

and so on. It follows from Eq.(2) that contributions to a given combinant from statistically independent distributions are additive. The utility of combinants in the analysis of experimental data was investigated by several authors $[4,5,6]$.

In a parallel development, observations of galaxy distributions have revealed the existence of dense regions where the matter density exceeds the average density of the universe by many orders of magnitude as well as regions devoid of matter [7]. A particularly useful quantity to investigate is the galactic void probability which can be used as a generating function for all the other probabilities [8]. It was also shown that the probabilities of having 
voids in the large-scale distributions of galaxies and rapidity gap probabilities in the multiplicity distributions of particles produced in hadron collisions exhibit similar scaling properties $[6,9,10]$.

Factorial cumulant moments, $f_{k}$, are defined by the generating function

$$
\log \left[\sum_{N}(1-\lambda)^{N} P_{N}\right]=\sum_{k=1}^{\infty} \frac{(-\lambda)^{k}}{k !} f_{k}
$$

and they can be written as the integrals of the cumulant correlation functions

$$
f_{k}=n^{k} \int_{V} d x_{1} \ldots d x_{k} \omega_{k}\left(x_{1}, \ldots, x_{k}\right)
$$

where $x_{1}, \ldots, x_{k}$ represent the quantity in which the correlation is measured (rapidity, galactic coordinate, etc.), $V$ is the range of allowed values of these quantities (rapidity bin, galactic sample volume,..) and $n=\langle N\rangle / V$ is the average number of particles in this range. Setting $\lambda=1$ in Eq. (6) White [8] and independently Mueller [11] have shown that the void probability can be written in terms of the integrals of $\mathrm{N}$-particle correlation functions

$$
W \equiv \log P_{0}=\sum_{k=1}^{\infty} \frac{(-n)^{k}}{k !} \int_{V} d x_{1} \ldots d x_{k} \omega_{k}\left(x_{1}, \ldots, x_{k}\right)
$$

One can easily write down [8] higher probabilities by differentiating $W$ with respect to $\mathrm{n}$ :

$$
P_{N}=\frac{(-n)^{N}}{N !} \frac{d^{N}}{d n^{N}} e^{W}
$$

Given that the galaxy correlation functions grow rather fast with increasing $k$ for the first few measured terms in the series in Eq. (8) it is difficult to evaluate void probability by summing this series. An alternative possibility is to write the void probability in terms of combinants using Eq. (2) :

$$
P_{0}=\exp \left[-\sum_{k} \mathcal{C}_{k}\right]
$$

It follows that for combinants one can write an expression similar to that given in Eq. (9) :

$$
\mathcal{C}_{N}=\frac{(-n)^{N}}{N !} \frac{d^{N}}{d n^{N}} W
$$


In medium-energy hadron collisions $\mathcal{C}_{k}$ decreases as $k$ increases $[4,5]$. Because of cancellations in Eqs. (3) through (5) one might expect this to be a rather general result and consequently the series in Eq. (8) to converge faster than the series in Eq. (8).

Factorial moments, $\zeta_{k}$, are defined by the generating function

$$
\sum_{N}(1-\lambda)^{N} P_{N}=\sum_{k=1}^{\infty} \frac{(-\lambda)^{k}}{k !} \xi_{k},
$$

can be written as the integrals of the density correlation functions

$$
\xi_{k}=\int_{V} d x_{1} \ldots d x_{k} \rho_{k}\left(x_{1}, \ldots, x_{k}\right)
$$

In the absence of correlations (i.e. for Poisson distribution) all the higher order density correlation functions can be written as a single product

$$
\rho_{k}\left(x_{1}, \ldots, x_{k}\right)=\rho_{1}\left(x_{1}\right) \rho_{1}\left(x_{2}\right) \ldots \rho_{1}\left(x_{k}\right) \text {. }
$$

\section{Models of Independent Particle Emission}

In this section we show that combinants are also useful in identifying the nature of the source of the emitted bosons, e.g., whether they are coherently emitted or come from the secondary decays of fermions using simplified models of independent emission. Let us first consider a configuration with $S$ sources emitting bosons without any restrictions on the number of bosons emitted by any source. Let us assume that $b_{i}$ is the unnormalized probability of emitting one boson (and $b_{i}^{N}$ is that of emitting $N$ bosons) by the $i$ th source. The total probability of emitting one boson is

$$
P_{1}^{S} \propto \sum_{i=1}^{S} b_{i}
$$

and that of emitting two bosons is

$$
P_{2}^{S} \propto \sum_{i, j=1}^{S} b_{i} b_{j}
$$


and so on. One observes that $P_{N}^{S}$ is the complete symmetric function [12] of degree $N$ in the arguments $b_{i}$. Using the generating function for complete symmetric functions, the generating function for properly normalized probabilities can be written as

$$
\begin{aligned}
\sum_{N=0}^{\infty} P_{N}^{S} \lambda^{N} & =\prod_{i=1}^{S}\left(\frac{1-b_{i}}{1-\lambda b_{i}}\right) \\
& =\exp \left[\sum_{i=1}^{S} \log \left(1-b_{i}\right)-\sum_{i=1}^{S} \log \left(1-\lambda b_{i}\right)\right]
\end{aligned}
$$

By expanding the logarithms one can show that, for $b_{i} \rightarrow 0$, Eq. (18) reduces to Eq. (1), i.e., the above distribution becomes Poisson. If we take emission probabilities from all the sources to be the same, $b_{i}=b$, then Eq. (17) reduces to the generating function of a negative binomial distribution:

$$
\iota^{\prime}(\lambda)=\left(\frac{1-b}{1-\lambda b}\right)^{s}
$$

which has been shown to reproduce the multiplicities data at energies below $\sqrt{s} \sim 100 \mathrm{GeV}$. The combinants for the distribution in Eq. (18) can be easily obtained by expanding the logarithm in a power series

$$
\mathcal{C}_{k}=\frac{1}{k} \sum_{i=1}^{S} b_{i}^{k}
$$

The quantities $b_{i}$ depend on the underlying physics and they should be determined from a given model. For boson pairs with transverse mass $\mu=$ $\sqrt{m^{2}+k_{\perp}^{2}}$ emitted from an external, static electric field $E$ one gets [13]

$$
b_{i}=\left[1+\exp \left(\frac{\pi \mu^{2}}{e E}\right)\right]^{-1},
$$

and for boson emission from a thermal source one has [13]

$$
b_{i}=\exp \left(-\beta_{i}\right)
$$

where $\beta$ is the inverse temperature and $\epsilon_{i}$ represent the energy levels available in the source. 
One should remark that Eq. (17) is related to the character generating formula for the symmetric group. It is not surprising that the problem of multiplicities for noninteracting particles is related to that of the representations of symmetric group, although the consequences of this relation are not yet fully studied.

Next we consider a configuration with $M$ sources, but this time each capable of emitting only a given number of bosons (typically one or two). Such a situation would be realized, for example, if these sources are particles decaying into bosons. If we take the source for the negative pions to be $\Delta^{0}$ particles, then such a source can either emit no $\pi^{-}$'s (when it decays into $\pi^{0}$ s) or one $\pi^{-}$. For definiteness in the following we shall assume that each source can emit at most one boson. A generalization to a more con uplicated situation is straightforward. Let us assume that $z_{i}$ is the relative probability of emitting one boson by the $i$ th source. The total probability of emitting one boson is

$$
P_{1}^{M} \propto \sum_{i=1}^{M} z_{i},
$$

and that of emitting two bosons is

$$
P_{2}^{M} \propto \sum_{i \neq j} z_{i} z_{j}
$$

and so on. This time $P_{N}^{M}$ is the elementary symmetric function of degree $N$ in the arguments $z_{i}$. (In the complete symmetric functions the powers of the same argument are allowed, in the elementary symmetric functions they are not [12]). The generating function for the properly normalized probabilities can be written as

$$
\begin{aligned}
\sum_{n=0}^{\infty} P_{N}^{M} \lambda^{n} & =\prod_{i=1}^{M}\left(\frac{1+\lambda z_{i}}{1+z_{i}}\right) \\
& =\exp \left[\sum_{i=1}^{M} \log \left(1+\lambda z_{i}\right)-\sum_{i=1}^{M} \log \left(1+z_{i}\right)\right] .
\end{aligned}
$$

Because of the restrictions on the number of bosons emitted from each source, one observes that the probability distribution function in Eq. (25) is like the probability distribution function of fermions. In fact, fermion pairs produced 
in background fields follow a very similar multiplicity distribution [14]. Introducing

$$
d_{i}=\frac{z_{i}}{1+z_{i}}
$$

one can rewrite $\mathrm{Eq} .(27)$ as

$$
\sum_{n=0}^{\infty} P_{n}^{M} \lambda^{n}=\exp \left\{\sum_{i=1}^{M} \log \left[1+(\lambda-1) d_{i}\right]\right\} .
$$

Again as $d_{i} \rightarrow 0$, the above distribution becomes Poisson. The combinants for the distribution in Eq. (27) can also be obtained by expanding the logarithm

$$
\mathcal{C}_{k}=(-1)^{k+1} \frac{1}{k} \sum_{i=1}^{M} z_{i}^{k}
$$

One observes that the combinants associated with the distribution of Eq. (28) alternate in sign as $k$ takes values $1,2,3, \ldots$ whereas the combinants associated with the distribution of Eq. (18) are always positive. Thus the sign of the combinants extracted from the multiplicities data could provide information on the mechanism responsible for the emission. An application of these ideas to the pion production in nuclear collisions was given in Ref. [5] where the reader is referred to for further details.

\section{$3 \quad$ Scaling Properties}

In this section we discuss the scale invariance properties of the moments. Assuming scale-invariance of many-body correlation functions,

$$
\omega_{k}\left(\lambda x_{1}, \ldots, \lambda x_{k}\right)=\lambda^{-(k-1) \gamma} \omega_{k}\left(x_{1}, \ldots, x_{k}\right),
$$

and introducing the scaled coordinates

$$
z_{k}=x_{k} / V, z_{k} \leq 1
$$

one can rewrite reduced factorial cumulants in terms of an arbitrarily chosen scale $V_{0}[15]$ :

$$
\zeta_{k} \equiv \frac{f_{k}}{\langle N\rangle^{k}}=\left(\frac{V_{0}}{V}\right)^{(k-1) \gamma} S_{k}
$$


where

$$
S_{k}=\int_{0}^{1} d z_{1} \ldots d z_{k} \omega_{k}\left(V_{0} z_{1}, \ldots, V_{0} z_{k}\right)
$$

In the equations above $S_{k}$ are c-numbers which depend only on the shapes of the higher order correlation functions $\left(S_{1}=1\right)$ and $\gamma$ is a parameter controling the large distance behavior of the two-body correlation function :

$$
\zeta_{2}=\left(\frac{V_{0}}{V}\right)^{\gamma}
$$

where the scale $V_{0}$ has been chosen so that $S_{2}=1$. The scaling properties displayed in Eq. (30) are more general than those assumed in factorized hierarchial models of galaxy distributions [7] or those assumed in the linkedpair ansatz models of rapidity correlation functions [16], but gives the same result as those models for the scaling of reduced factorial cumulants (eq. (32)). Eq. (32) implies a remarkable result for the scaled void probability, $\chi$,

$$
\lambda \equiv-\log P_{0} /<N>
$$

namely, that it depends only on the combination $\langle N\rangle \zeta_{2}$ :

$$
\chi=\chi\left(<N>\zeta_{2}\right)=V \sum_{k=1}^{\infty}\left(-<N>\zeta_{2}\right)^{(k-1)} \frac{S_{k}}{k !} .
$$

Note that one can derive all the probabilities from Eq. (36) using Eq. (9). For all these probabilities to satisfy the condition $0 \leq P_{n} \leq 1$, and for the series in Eq. 36 ) to be convergent all the $S_{k}$ should be bounded from above (implying a maximal correlation), or alternately the function $\chi\left(<N>\zeta_{2}\right.$ ) should be bounded from below. It is interesting to note that the observed galaxy distributions lie very close to this "unitarity bound" [9], i.e. galaxies are clustered almost maximally.

Using Eq. (11) one can easily show that, the scaling property depicted above implies not only the entire sum in Eq. (8), but each term in that sum seperately scales :

$$
\mathcal{C}_{k}=\left\langle N>c_{k}\left(<N>\zeta_{2}\right),\right.
$$

indicating once again the suitability of Eq. (10) in evaluating galactic void distributions and rapidity gap probabilities. The series in the exponential of Eq. (10) remains scale invariant no matter at which order it is truncated. 
Finally one should note that if the density correlation functions, given in Eq. (13), scale like in Eq. (30), i.e.

$$
\rho_{k}\left(\lambda y_{1}, \ldots, \lambda y_{k}\right)=\lambda^{f_{k}} \rho_{k}\left(y_{1}, \ldots, y_{k}\right),
$$

the resulting behavior of the reduced factorial moments is called intermittency [17]:

$$
\frac{<N(N-1) \ldots(N-i+1)>}{<N>^{i}} \sim(Y)^{-f_{i}}
$$

where $Y$ is the rapidity bin size.

This research was supported in part by U.S. National Science Foundation Grants PHY-9314131 and IN'T-9001308 and in part by Department of Energy. The author acknowledges the gracious hospitality of the members of Institute for Nuclear Theory at University of Washington where part of the research leading to this work was performed.

\section{References}

[1] K.L. Wieand, S.E. Pratt, and A.B. Balantekin, Phys. Lett. B 274, 7 (1992).

[2] J.D. Bjorken and S.D. Drell, Relativistic Quantum Fields (McGraw-Hill, New York, 1965), pp. 202-207.

[3] S.K. Kauffmann and M. Gyulassy, J. Phys. A : Math. Gen. 11, 298 (1978).

[4] J. Bartke, Phys. Scripta 27, 225 (1983).

[5] A.B. Balantekin and J.E. Seger, Phys. Lett. B266, 231 (1991).

[6] S. Hegyi, Phys. Lett. B 309, 443 (1993).

[7] P.J.E. Peebles, The Large Scale Structure of the Universe (Princeton University Press, Princeton, 1980).

[8] S.D.M. White, Monthly Notices Roy. Astron. Soc. 186, 145 (1979).

[9] J. Fry, R. Giovanelli, M.P. Haynes, A. Melott and R.J. Scherrer, Astrophys. J. 340, 11 (1989). 
[10] P. Carruthers and C.C. Shih, Phys. Lett. 127B, 242 (1983).

[11] A.H. Mueller, Phys. Rev. D4, 150 (1971).

[12] I.G. Macdonald, Symmetric Functions and Hall Polynomials (Oxford University Press, Oxford, 1979).

[13] A.B. Balantekin, in Proc. of the International Symp. on Group Theory and Special Symmetries in Nuclear Physics, Ann Arbor 1991, J. Draayer, Ed., p.16 (World Scientific, Singapore, 1992).

[14] A.B. Balantekin, S.H. Fricke, and J.E. Seger, Int. J. Mod. Phys. A 6, 695 (1991).

[15] R. Balian and R. Schaeffer, Astron. Astrophys. 220, 1 (1989).

[16] P. Carruthers and I. Sarcevic, Phys. Rev. Lett. 63, 1562 (1989).

[17] A. Bialas and R. Peschanski, Nucl. Phys. B273, 703 (1986). 

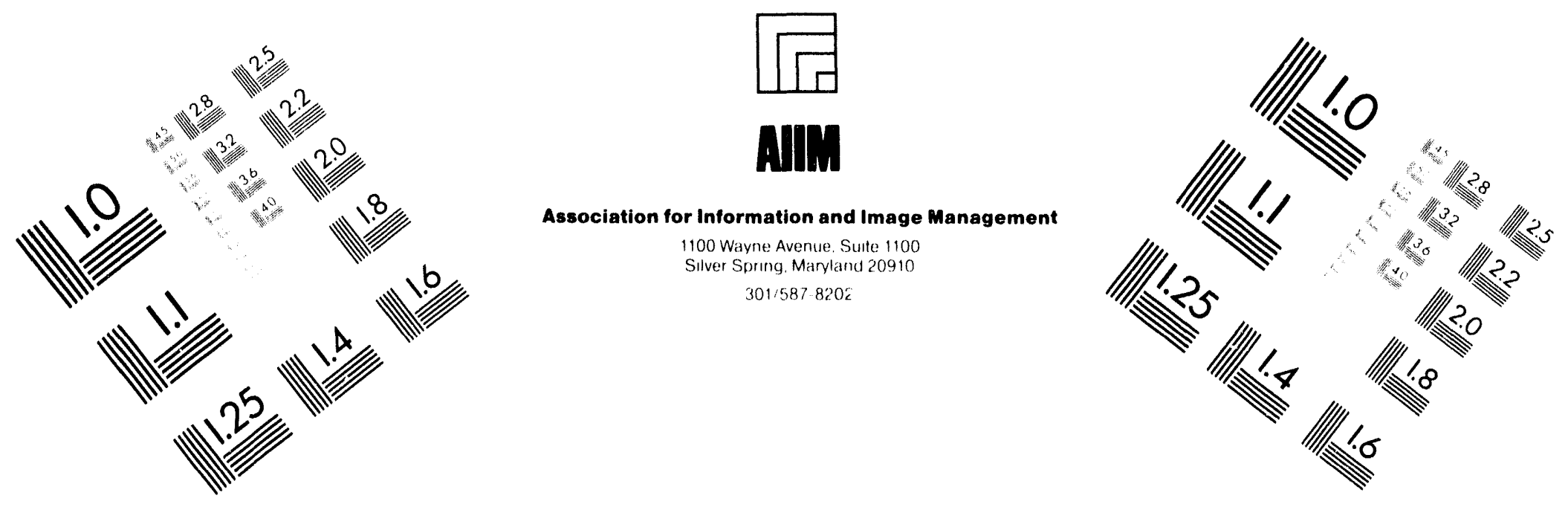

\section{Centimeter}

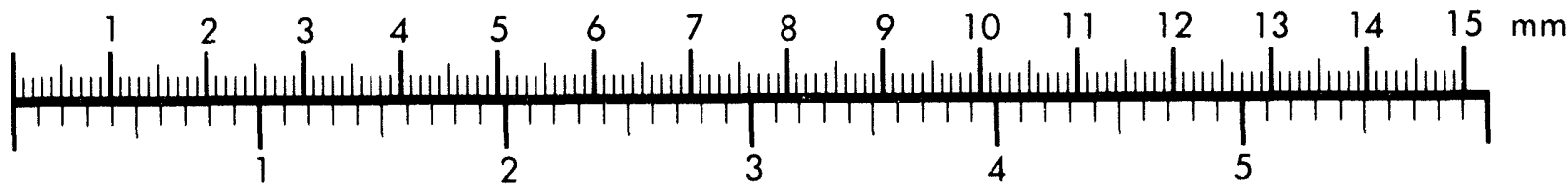

Inches
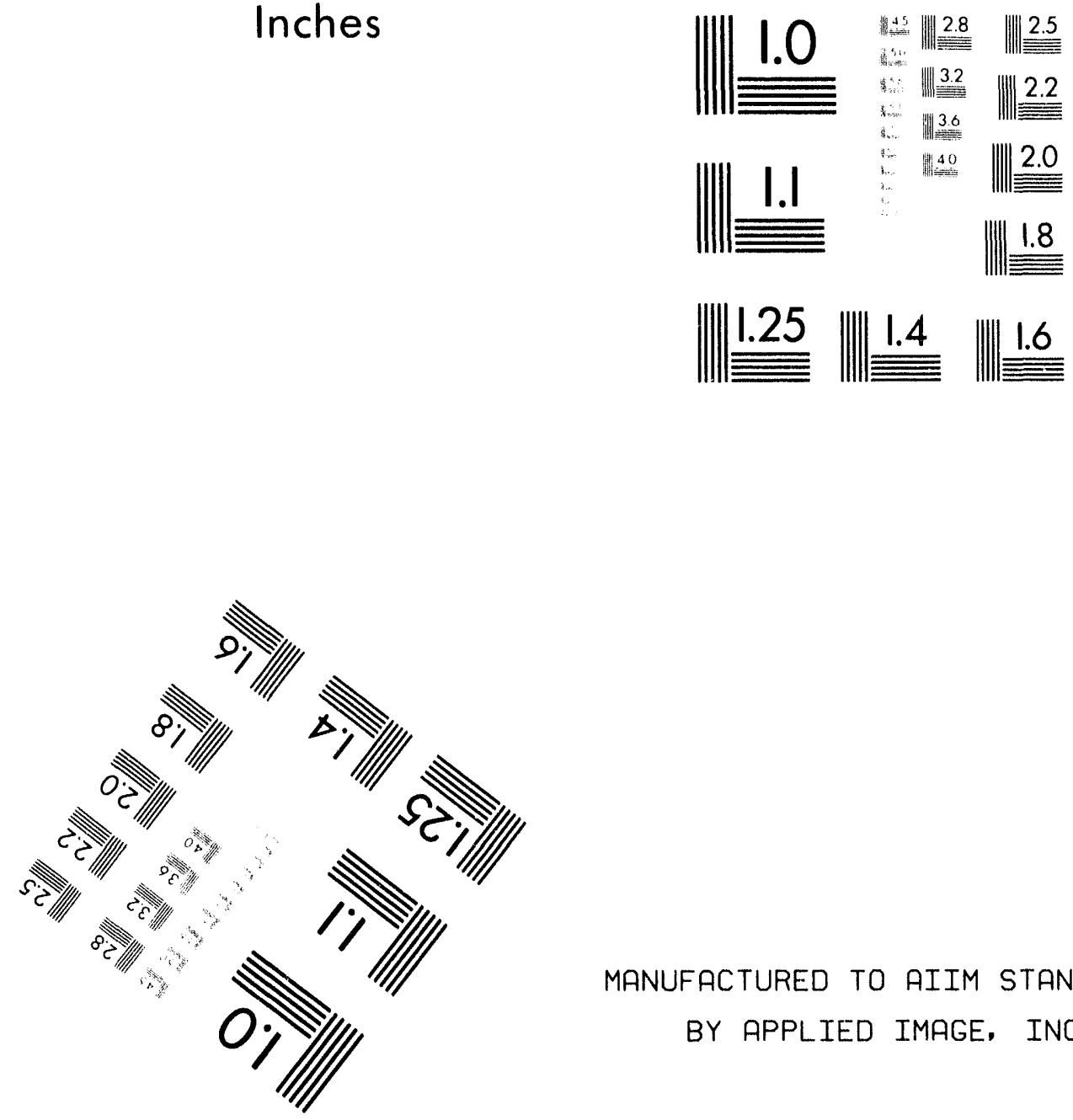

MANUFACTURED TO AIIM STANDARDS

BY APPLIED IMAGE. INC.

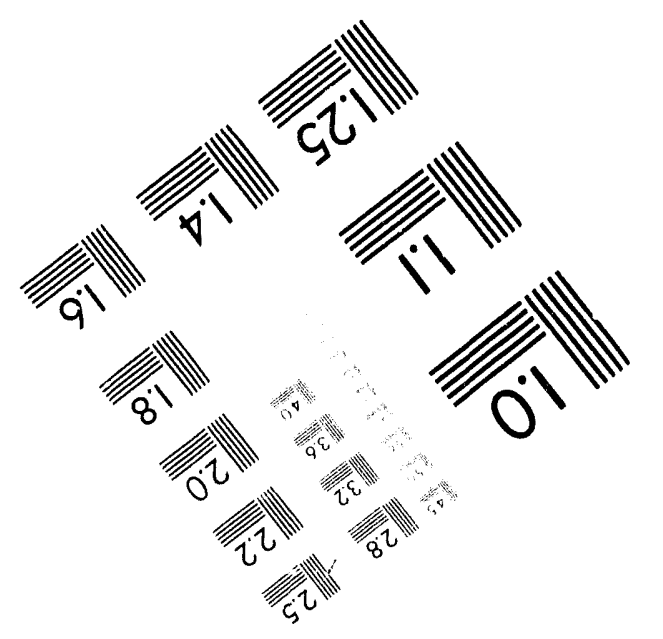



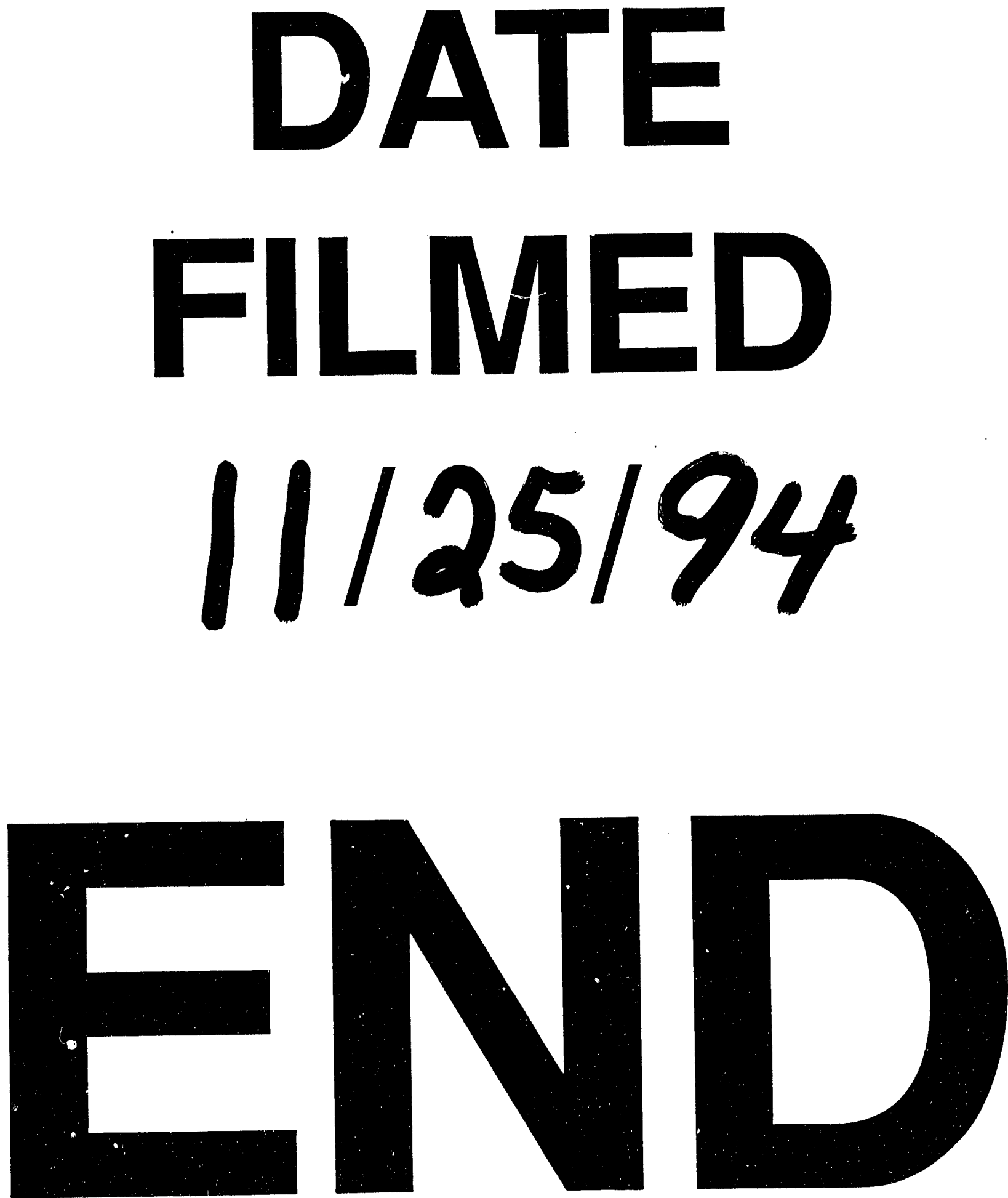